\title{
ESTUDIO SOBRE LA AUSENCIA DE NORMATIVIDAD LEGAL EN LA ADQUISICIÓN DEL SOAT (SEGURO OBLIGATORIO DE ACCIDENTES DE TRÁNSITO) EN CIUDADANOS EXTRANJEROS EN LA REGIÓN TACNA.
}

\author{
STUDY ON THE ABSENCE OF LEGAL STANDARDS IN THE ACQUISITION OF SOAT (COMPULSORY INSURANCE \\ TRAFFIC ACCIDENT) FOR FOREIGN CITIZENS IN THE REGION TACNA.
}

\author{
Herminia Sarmiento Chambi ${ }^{1}$
}

\begin{abstract}
RESUMEN
Objetivo: Determinar la incidencia de la normatividad en la adquisición obligatoria del SOAT por extranjeros en la protección y seguridad de las víctimas de accidentes de tránsito.

Método: El tipo de investigación es relacional, de naturaleza descriptiva relacional, debido a que está basado en medir las propiedades de las variables a estudio: normatividad de adquisición del SOAT por extranjeros; y, protección y seguridad de las víctimas de accidentes de tránsito. Se adoptó el diseño (no experimental) descriptivo relacional, puesto que no se manipula la variable independiente; y se percibe medir la relación directa existente entre dos o más conceptos o variables.
\end{abstract}

Resultado: Se analizaron e interpretaron la información procesada a través del análisis cuantitativo, cualitativo, y la síntesis, determinándose la incidencia de la normatividad en la adquisición obligatoria del SOAT por extranjeros en la protección y seguridad de las víctimas de accidentes de tránsito. Conclusiones: En la Región Tacna, la protección y seguridad de las víctimas de accidentes de tránsito es deficiente.

En la Región Tacna, la aplicación de la normatividad de adquisición del SOAT por extranjeros es inadecuada, al encontrase que la regulación de la norma, el modo de aplicación, la garantía observada, impunidad; y el elevado porcentaje de accidentes de tránsito que se observa en el período 2011 -2012 por parte de extranjeros no permiten la eficiencia en su aplicación.

\section{PALABRA CLAVE}

Normatividad de adquisición del SOAT por extranjeros, Protección y seguridad, víctimas de accidentes de tránsito, medidas de prevención, eficiencia.

\section{ABSTRACT}

Objective: Determine the impact of regulations on the compulsory acquisition of SOAT by foreigners in the protection and safety of victims of traffic accidents.

Method: The research is relational, relational descriptive nature, because it is based on measuring the properties of the study variables: SOAT regulations acquisition by foreigners; and safety and security of victims of traffic accidents. Design (not experimental) relational description was adopted as the independent variable is not manipulated; is perceived and measured the direct relationship between two or more concepts or variables.

Result: Were analyzed and interpreted the information processed through quantitative, qualitative analysis, and synthesis, determining the impact of the regulations on compulsory acquisition of SOAT by foreigners in the protection and safety of victims of traffic accidents.

Conclusions: In the Tacna Region, the protection and safety of victims of traffic accidents is poor.

In the Tacna Region, the implementation of regulations SOAT acquisition by foreigners is inadequate, after finding that the regulation of the standard application mode, the observed guarantee impunity; and the high percentage of traffic accidents observed in the period $2011-2012$ by foreigners do not allow efficient implementation.

\section{KEYWORDS}

Acquisition Regulations SOAT by foreigners, Safety and security, victims of traffic accidents, prevention, efficiency.

1. Doctor en Derecho, Magister en Derecho con Mención en Ciencias Penales, Abogada. Conciliador Extrajudicial y de Familia. Fundador y Miembro de la Revista "KAMISARAKI". Socia Honoraria del "INTI DE AMERICA". Actualmente Labora en la Subdirección de Transporte Terrestre - DTT de la Dirección Regional de Transportes y Comunicaciones de Tacna - Especialista en Transporte Terrestre de Personas y Mercancía. 


\section{Introducción}

El seguro en el más estricto sentido repara el daño causado al asegurado, pero en el caso del SOAT no sólo al asegurado y a los ocupantes de un vehículo, sino también a los peatones que pudieran verse afectados por un accidente de tránsito, en atención a su esencia netamente social. En el caso del SOAT el beneficiario es la sociedad en su conjunto.

EI SOAT es un seguro obligatorio establecido por Ley con un fin netamente social. Su objetivo es asegurar la atención, de manera inmediata e incondicional, de las víctimas de accidentes de tránsito que sufren lesiones corporales y muerte. La frecuencia con que estos hechos de tránsito ocurren es alarmante y ha enlutado a muchas familias tacneñas, dejando huérfanos y viudas sin ningún apoyo económico; también ha provocado lesiones severas, dejando secuelas, incapacidad parcial 0 total en un número significativo de personas, en razón de esta problemática que acontece en la Región Tacna, y el porcentaje de accidentes de tránsito ocasionados por vehículos extranjeros. Por otro lado, para entender el aporte del SOAT es necesario conocer su naturaleza desde el ámbito social y económico; teniendo presente que los riesgos del accidente de tránsito está latente y la atención de las posibles víctimas debe actuarse de manera inmediata. Por lo tanto, el seguro es un valioso recurso de protección, en virtud de que la vida es el tesoro más preciado y a su vez el Seguro es considerado como un bienestar social y una actividad social; a su vez es catalogada como una prestación del servicio social y una viabilidad económica. También hay que tener en cuenta el principio de responsabilidad objetiva para lo cual hay que concientizar un cambio de actitud a los conductores y/o propietarios de vehículos; a efectos de que conduzcan con responsabilidad, para así prevenir los accidentes de tránsito que cada día se vienen incrementando en nuestro país, tanto por vehículos con placa de rodaje nacional y extranjera; la problemática descrita ha permitido la inquietud del presente trabajo de investigación denominado: "ESTUDIO SOBRE LA AUSENCIA DE NORMATIVIDAD LEGAL EN LA ADQUISICION DEL SOAT (SEGURO OBLIGATORIO DE ACCIDENTES DE TRANSITO) EN CIUDADANOS EXTRANJEROS EN LA REGION TACNA. 2011 2012".

\section{Materiales y métodos}

El tipo de investigación es relacional, de naturaleza descriptiva relacional, se adoptó el diseño (no experimental) descriptivo relacional, puesto que no se manipula la variable independiente; y se percibe medir la relación directa existente entre dos 0 más conceptos 0 variables.

Las unidades de estudio están constituidas por: Aseguradoras, Policías de División de Tránsito y Comisarías, Policías de Protección de Carreteras y las estadísticas de accidentes de tránsito por vehículos.

La población y muestra de estudio estuvo constituida por:

Tabla 1. Población y muestra

\begin{tabular}{|l|r|r|}
\hline Población & \multicolumn{1}{|c|}{ Número } & \multicolumn{1}{|c|}{ Muestra } \\
\hline Aseguradoras & 04 & 04 \\
\hline $\begin{array}{l}\text { Policías de la Región de } \\
\text { Tacna }\end{array}$ & 109 & 85 \\
\hline $\begin{array}{l}\text { Estadísticas de } \\
\text { Accidentes de Tránsito }\end{array}$ & 74 & 74 \\
\hline TOTAL & 04 & 04 \\
\hline
\end{tabular}

Para la recolección de datos se recurrió a la aplicación de los instrumentos de medición del cuestionario, la cédula de entrevista; y, la Ficha de análisis documental. Como técnicas para el desarrollo de la investigación se utilizaron: la encuesta, la entrevista; y, el análisis documental.

Desde el punto de vista geográfico el presente trabajo de investigación es a nivel Tacna, porque trata del estudio sobre la ausencia de normatividad legal en la adquisición del SOAT por ciudadanos extranjeros en la protección y seguridad de las víctimas de accidentes de tránsito.

La información se procesó electrónicamente, utilizando para ello el programa Microsoft Excel versión XP bajo ambiente Windows, SPSS (versión 18) para presentar los Ítems mediante gráficas $y$, al mismo tiempo estimar las frecuencias absolutas y relativas de cada reactivo para describir la variable a estudio. 


\section{Resultados}

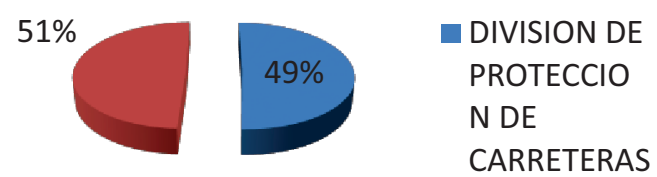

Figura 1

Tipo de Dependencia

El $51 \%$ de los policías pertenecen a la Dirección de Tránsito en Tacna; y, el 49\% a la División de Protección de Carreteras.

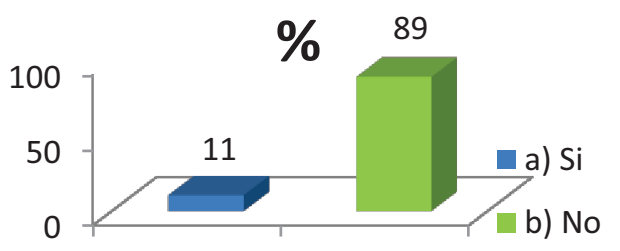

a) $\mathrm{Si}$

b) $\mathrm{No}$

Figura 2

La Policía Nacional de Carreteras tiene competencia en exigir el SOAT a los extranjeros que ingresan a Tacna en forma temporal:

El $89 \%$ de los policías manifiesta que la Policía Nacional de Carreteras "no" tiene competencia en exigir el SOAT a los extranjeros que ingresan a Tacna en forma temporal; y, sólo el $11 \%$ señala que si la tiene.

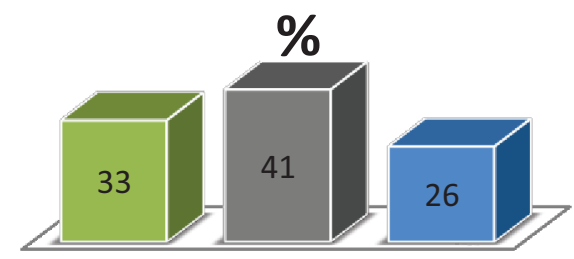

a) Atropello b) Choquec) Volcadura

Figura 3

Tipo de accidentes se han producido hasta la fecha de parte de los vehículos extranjeros que ingresan en forma temporal:

El 41\% manifiestan que los "choques" son el tipo de accidentes que han producido hasta la fecha los vehículos extranjeros que ingresan en forma temporal, el $33 \%$ que son los atropellos; $y$, el $26 \%$ señala que son las volcaduras.

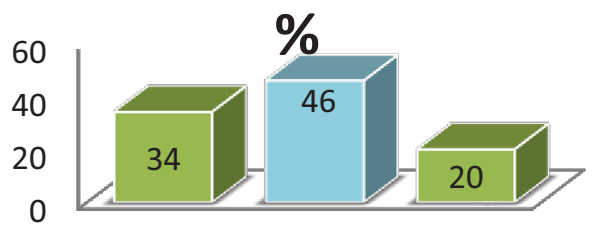
a) Alto
b) Medio
c) Bajo

Figura 4

Nivel porcentual de accidentes de tránsito

El $46 \%$ señalan que los accidentes de tránsito generado por vehículos extranjeros que ingresan en forma temporal, alcanzan el nivel porcentual "medio"; y el 34\% señala que es alto.

\section{$\%$}

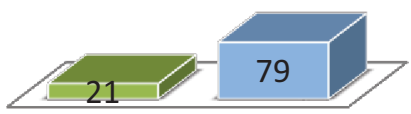

$\begin{array}{ll}\text { a) } \mathrm{Si} & \text { b) No }\end{array}$

Figura 5

Las medidas de protección y seguridad se encuentran reguladas adecuadamente para garantizar la seguridad física de las personas:

El $79 \%$ de los policías manifiestan que las medidas de protección y seguridad "no" se encuentran reguladas adecuadamente para garantizar la seguridad física de las personas; $y$, sólo el $21 \%$ señala lo contrario.

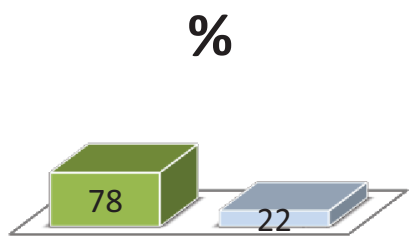

$\begin{array}{ll}\text { a) } \mathrm{Si} & \text { b) No }\end{array}$

Figura 6

La Ley General de Transporte y Tránsito Terrestre No. 27181, presenta un vacío al no reglamentar la obligatoriedad del SOAT para extranjeros:

El $78 \%$ de los policías manifiestan que la Ley General de Transporte y Tránsito Terrestre No. 27181, presenta un vacío al no reglamentar la obligatoriedad del SOAT para extranjeros; y, en menor frecuencia señalan lo contrario. 


\section{$\%$}

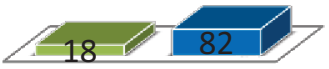

$\begin{array}{lll}\text { a) } \mathrm{Si} & \text { b) } \mathrm{No}\end{array}$

Figura 7

La Ley General de Transporte y Tránsito Terrestre No. 27181, permite indemnizar a las víctimas de accidentes de tránsito por vehículos extranjeros:

El $82 \%$ manifiestan que la Ley General de Transporte y Tránsito Terrestre No. 27181, no permite indemnizar a las víctimas de accidentes de tránsito por vehículos extranjeros.

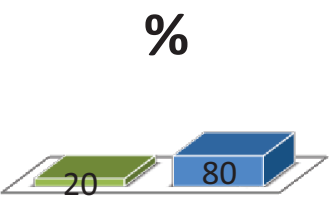

$\begin{array}{lll}\text { a) } \mathrm{Si} & \text { b) No }\end{array}$

Figura 8

La Ley General de Transporte y Tránsito Terrestre No. 27181, permite brindar atención médica a las víctimas de accidentes de tránsito por vehículos extranjeros:

El $80 \%$ manifiestan que la Ley General de Transporte y Tránsito Terrestre No. 27181, no permite brindar atención médica a las víctimas de accidentes de tránsito por vehículos extranjeros.

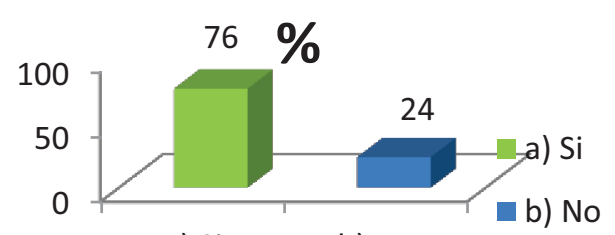

a) $\mathrm{Si}$

b) No

Figura 9

La ausencia de normatividad obligatoria sobre el SOAT para extranjeros permite la impunidad e incremento de accidentes de tránsito en la Región Tacna:

El $76 \%$ manifiestan que la ausencia de normatividad obligatoria sobre el SOAT para extranjeros permite la impunidad e incremento de accidentes de tránsito en la Región Tacna.

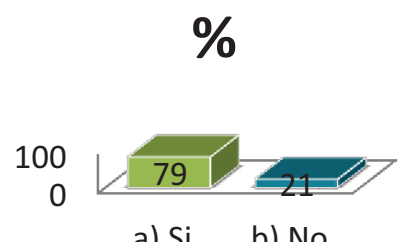

$\begin{array}{lll}\text { a) } \mathrm{Si} & \text { b) No }\end{array}$

Figura 10

La falta de obligatoriedad del Seguro contra accidentes de tránsito provocados por extranjeros no permite proteger e indemnizar los daños y perjuicios a las víctimas de accidentes de tránsito:

El $79 \%$ manifiestan que la falta de obligatoriedad del Seguro contra Accidentes de Tránsito provocados por extranjeros no permite proteger e indemnizar los daños y perjuicios.

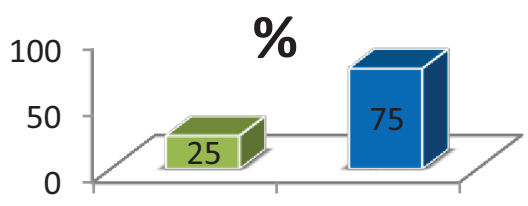

a) $\mathrm{Si}$

b) No

Figura 11

La actual Ley General de Transporte y Tránsito Terrestre No. 27181, permite la protección y seguridad de las víctimas de accidentes de tránsito por extranjeros:

El 75\% manifiestan que la actual Ley General de Transporte y Tránsito Terrestre No. 27181, no permite la protección y seguridad de las víctimas de accidentes de tránsito por extranjeros.

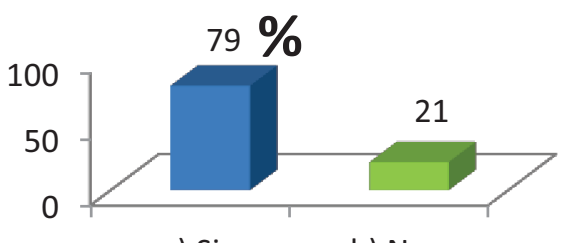
a) $\mathrm{Si}$
b) No

Figura 12

Se debe regular la obligatoriedad de adquirir SOAT de parte de los extranjeros a fin de poder brindar protección y seguridad a las víctimas de accidente de tránsito:

El $79 \%$ manifiestan que se debe regular la obligatoriedad de adquirir SOAT de parte de los extranjeros. 


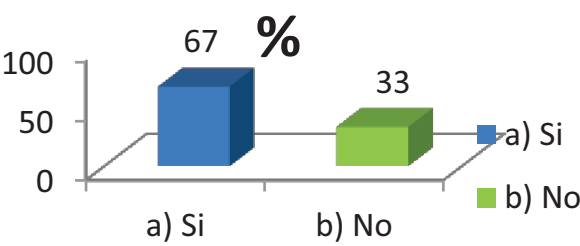

Figura 13

La incorporación de la aplicación de la normatividad de adquisición del SOAT por extranjeros, garantizaría que las víctimas de accidentes de tránsito tengan accesos a servicios de salud y adecuados tratamientos para su restablecimiento:

El $67 \%$ de los policías manifiestan que la incorporación de la aplicación de la normatividad de adquisición del SOAT por extranjeros, garantizaría que las víctimas de accidentes de tránsito tengan accesos a servicios de salud y adecuados tratamientos para su restablecimiento.

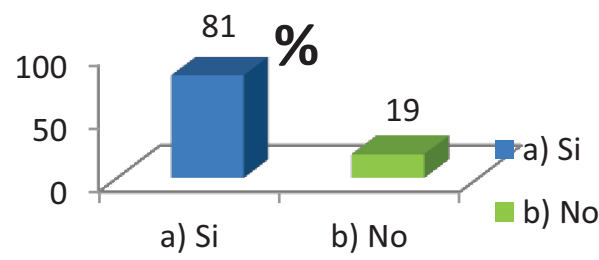

Figura 14

La incorporación de la aplicación de la normatividad de adquisición del SOAT por extranjeros, debe permitir también indemnizar a sus beneficiarios por concepto de incapacidad temporal, invalidez y muerte:

El $81 \%$ de los policías manifiestan que la incorporación de la aplicación de la normatividad de adquisición del SOAT por extranjeros, debe permitir también indemnizar a sus beneficiarios por concepto de incapacidad temporal, invalidez y muerte.

\section{Discusión}

La presente investigación determinó la inadecuada aplicación de la normatividad de adquisición del SOAT por extranjeros y su incidencia desfavorable en la protección y seguridad de víctimas de accidentes de tránsito, período 2011-2012; ya que se comprobó que en la Región Tacna la aplicación de la normatividad de adquisición del SOAT por extranjeros es inadecuada; y, la protección y seguridad de las víctimas de accidentes de tránsito es deficiente.

EI SOAT es un seguro obligatorio establecido por Ley con un fin netamente social. Su objetivo asegurar la atención, de manera inmediata e incondicional, de las víctimas de accidentes de tránsito que sufren lesiones corporales y muerte, y de esta manera brindarles las medidas de protección pertinentes para garantizarles la atención y recuperación necesaria.

En congruencia la Universidad de los Andes, en el Informe Final sobre "Estudio Seguro Obligatorio de Responsabilidad Civil por Accidentes de Tránsito en la República de Colombia elaborado para Fasecolda, precisa que analizados todos los presupuestos establecidos en nuestro ordenamiento sobre la responsabilidad civil extracontractual por accidentes de tránsito y su cobertura, es claro que el establecimiento del seguro obligatorio de responsabilidad civil por accidentes de tránsito, desde el punto de vista jurídico, resulta conveniente; dado que, actualmente el sistema de cobertura establecido no abarca la totalidad de los perjuicios que se pueden generar a partir de la ocurrencia de un accidente de tránsito. Ahora, si bien el establecimiento del seguro obligatorio de responsabilidad civil por accidentes de tránsito resulta de gran interés general, es necesario tener en cuenta que el mismo, dada su naturaleza de seguro obligatorio, implica ciertos requisitos y características, como son: Debe ser un seguro impuesto por la Ley: "Solamente por Ley podrán 
crearse seguros obligatorios". Por lo tanto, para el establecimiento del seguro obligatorio de responsabilidad civil por accidentes de tránsito, es necesario la expedición de una Ley con todas sus formalidades por virtud de la cual se imponga el mismo".

Adicionalmente, Henry Fabián Camatón Vélez (2011) en la tesis titulada: COBERTURA DEL SOAT EN LOS ACCIDENTES DE TRÁNSITO EN LA PROVINCIA DE SANTA ELENA 2010 2011, manifiesta lo siguiente:

Las personas que sufren de un accidente de tránsito son aquellas quienes gozan de este servicio, Seguro Obligatorio de Accidentes de Tránsito (SOAT) la cual está amparado en la Constitución de la República del Ecuador y otras leyes, por ello la presente tesis tiene como objetivo primordial llegar a la conciencia de los integrantes del FONSAT y las Aseguradoras de este Servicio que son quienes cumplen en cubrir los gastos médicos y de hospitalización cuando un accidentado ingresa a una casa de salud.

La presente investigación argumenta que estos resultados también son una problemática que debe constituir preocupación por las autoridades competentes en la Región Tacna; y de esta manera poder mejorar la aplicación de la normatividad de adquisición del SOAT por extranjeros y la eficiente protección y seguridad a las víctimas de accidentes de tránsito por vehículos extranjeros.

\section{Referencias Bibliográficas}

- Universidad de los Andes (2006). Estudio Seguro Obligatorio de Responsabilidad Civil por Accidentes de Tránsito. 2006. Bogotá.

- Camatón, H. (2011). Cobertura del SOAT en los Accidentes de Tránsito en la Provincia de Santa Elena 2010-2011. Universidad Estatal Península de Santa Elena, Ecuador.

Email: hesarch@hotmail.com

Recibido: 06/11/2014

Aceptado para publicación: $12 / 12 / 2014$ 